Accelerator Division

Alternating Gradient Synchrotron Department

BROOKHAVEN NATIONAL LABORATORY

Upton, New York 11973

Accelerator Division

Technical Note

AGS/AD/Tech. Note No. 409

\title{
BARRIER CAVITY LONGITUDINAL DYNAMICS
}

\author{
M. Blaskiewicz
}

January 13,1995

\section{Introduction}

During the 1994 proton run a slow loss observed on the AGS injection porch severely limited the number of protons that could in principle be accelerated. This loss was drastically reduced when the RF was turned off and, to a lesser extent, when the bunch length was increased. During heavy ion operation the intensity per bunch was limited by devices upstream of the AGS.

A barrier cavity system in the AGS might increase the intensity for both these situations. This Note serves as a general introduction to the subject with AGS specific examples.

\section{Definitions and General Considerations}

Before proceeding it will be useful to define some relevant coordinates. The azimuth $\theta$ refers to longitudinal position with respect to the physical machine and increases by $2 \pi$ each turn. For a coasting beam with no collective effects I assume $\dot{\theta}=\omega(E, B)$ where the dot means time derivative and the angular frequency is a function only of particle energy, $E$, and (dipolar) magnetic field, $B$. For the sake of simplicity I will assume that the RF field is limited to a negligibly narrow range around $\theta=0$. The effect of several gaps will be similar as long as the time delays of the fields are correct. The RF system creates an electric field whose component along the beam velocity is given by $V(t) \delta_{p}(\theta) / R$ where $V(t)$ is the gap voltage as a function of time, $R$ is the 
machine radius and

$$
\delta_{p}(\theta)=\sum_{k=-\infty}^{\infty} \delta(\theta-2 \pi k)
$$

is the periodic delta function. I assume a constant magnetic field and an ideal energy $E_{0}$ with corresponding angular frequency $\omega_{0}$. A comoving coordinate is defined by the relative phase $\phi=\theta-\omega_{0} t$. Let $\phi_{n}$ and $E_{n}$ correspond to the relative phase and the total energy a particle has just after traversing the cavity for the $n$th time. To leading order and neglecting collective effects,

$$
\begin{gathered}
\phi_{n+1}-\phi_{n}=-\frac{2 \pi \eta\left(E_{n}-E_{0}\right)}{\beta^{2} E_{0}} \\
E_{n+1}-E_{n}=q V\left(n T_{0}-\phi_{n+1} / \omega_{0}\right)
\end{gathered}
$$

where $q$ is the charge of the particle, $\eta$ is the frequency slip factor, $\beta=v / c$ and $T_{0}$ is the revolution period.

Though the final calculations will require the difference equations, it will be useful to consider their continuum limit. Let $n \equiv \theta / 2 \pi$ be the time-like variable. Equations (1) and (2) are exactly equivalent to

$$
\begin{gathered}
\frac{d \phi}{d n}=-\frac{2 \pi \eta\left(E-E_{0}\right)}{\beta^{2} E_{0}} \\
\frac{d E}{d n}=q 2 \pi \delta_{p}(2 \pi n) V\left(n T_{0}-\phi / \omega_{0}\right) .
\end{gathered}
$$

For $|\phi|<\pi$,

$$
V\left(t-\phi / \omega_{0}\right)=\sum_{k=-\infty}^{\infty} a_{k}(t) e^{-i k\left(\phi-\omega_{0} t\right)},
$$

where the Fourier coefficients are given by

$$
a_{k}(t)=\int_{t-T_{0} / 2}^{t+T_{0} / 2} \frac{d x}{T_{0}} V(x) e^{-i k \omega_{0} x}
$$

Expanding the periodic delta function as a Fourier transform and extracting the diagonal terms in the double sum of Fourier coefficients gives,

$$
\begin{aligned}
& \frac{d \phi}{d n}=-\frac{2 \pi \eta\left(E-E_{0}\right)}{\beta^{2} E_{0}} \\
& \frac{d E}{d n}=q \hat{V}\left(n T_{0}, \phi / \omega_{0}\right) .
\end{aligned}
$$


The smoothed voltage $\hat{V}(t, \phi)$ is given by the Fourier series

$$
\hat{V}(t, \phi)=\sum_{k=-\infty}^{\infty} a_{k}(t) e^{-i k \phi}
$$

For a strictly periodic voltage with $V(t)=V\left(t+T_{0}\right)$ the Fourier coefficients do not depend on $t$ and the smoothed voltage is given by $\hat{V}(t, \phi)=V\left(-\phi / \omega_{0}\right)$. Equations (7) and (8) are generated by the Hamiltonian

$$
H(\phi, E)=-\frac{\pi \eta\left(E-E_{0}\right)^{2}}{\beta^{2} E_{0}}+U\left(n T_{0}, \phi\right),
$$

where $\phi$ is the coordinate variable and $E$ is its conjugate momentum. The potential is given by

$$
U(t, \phi)=-q \int_{0}^{\phi} \hat{V}(t, x) d x
$$

As long as $U(t, \phi)$ varies smoothly enough, the adiabatic invariant given by $J=\oint E d \phi$, where the integral is over one period of the motion, will be nearly conserved. Additionally, the Hamiltonian approximation predicts that $H(E, \phi)$ will be constant when the coefficients $a_{k}(t)$ are independent of time. A system which has the capability of conserving the adiabatic invariant will allow greater control over the phase space evolution.

Before proceeding to the general case where $\hat{V}(t, \phi)$ has explicit time dependence, consider the purely periodic case. As is well known, the standard map with $\overline{F(\phi)}=-V_{0} \sin \phi$ always shows some chaos when evolved by equations (1) and (2) while the associated Hamiltonian system is integrable. Experience with simulations shows that the difference is usually not: too important for smojoth forces but when the voltage is not smooth the difference can be considetable. For example, consider the extreme case where. the voltage has the form

$$
V(\phi)=\left\{\begin{array}{cll}
V_{0}, & \text { if } & 0<\phi<\phi_{0} \\
0, & \text { if } & \phi_{0}<\phi<2 \pi-\phi_{0} \\
-V_{0} & \text { if } & 2 \pi-\phi_{0}<\phi<2 \pi
\end{array}\right.
$$

and the voltage is defined globally via periodic extension. For definiteness I take $\eta<0$ and assume that $q V_{0} \phi_{0}>H(E, \phi)$ for energies of interest. In 


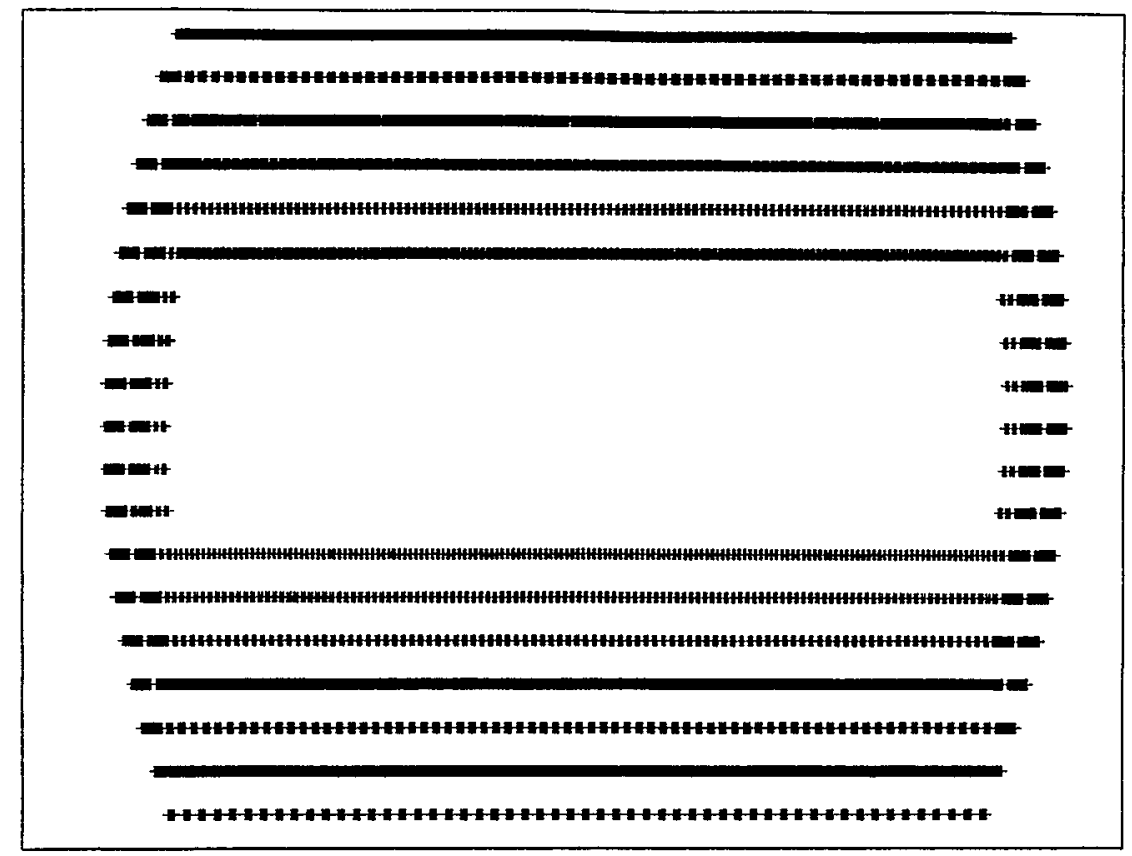

Figure 1: Energy deviation versus relative phase plotted once per turn. The particle either jumps one energy level on a given turn or its energy remains unchanged.

the Hamiltonian approximation $H(E, \phi)$ is constant for a given particle as it bounces back and forth. Evolution using equations (1) and (2) is different. When a particle bounces off the side of the well it is kicked an integer number of times where each energy kick has magnitude $q V_{0}$. As shown in Figure 1, the motion can be quite irregular even though the instantaneous synchrotron tune is fairly small. This result is due to the fact that the restoring voltage is discontinuous and the diagonal approximation used in obtaining the smooth Hamiltonian (10), is not good. In a real machine it would probably be difficult to create a situation where the sort of dynamics shown in Figure 1 would matter, though it seems prudent that voltage waveforms be studied numerically to be sure.

\section{Single Particle Barrier Dynamics}

In this -section the effect of changing the shape of the voltage waveform is studied. For simplicity it is assumed that the cavity voltage is a sum of the form

$$
V(t)=V_{1} G\left(t / T_{0}-\tau_{1}\right)+V_{2} G\left(t / T_{0}-\tau_{2}\right)
$$

where the parameters $V_{j}$ and $\tau_{j}$ vary (slowly) with time and

$$
G(s)=\left\{\begin{array}{lll}
\sin \pi(s-[s]) / w, & \text { if } & 0<\pi(s-[s]) / w<2 \pi \\
0, & \text { otherwise. }
\end{array}\right.
$$




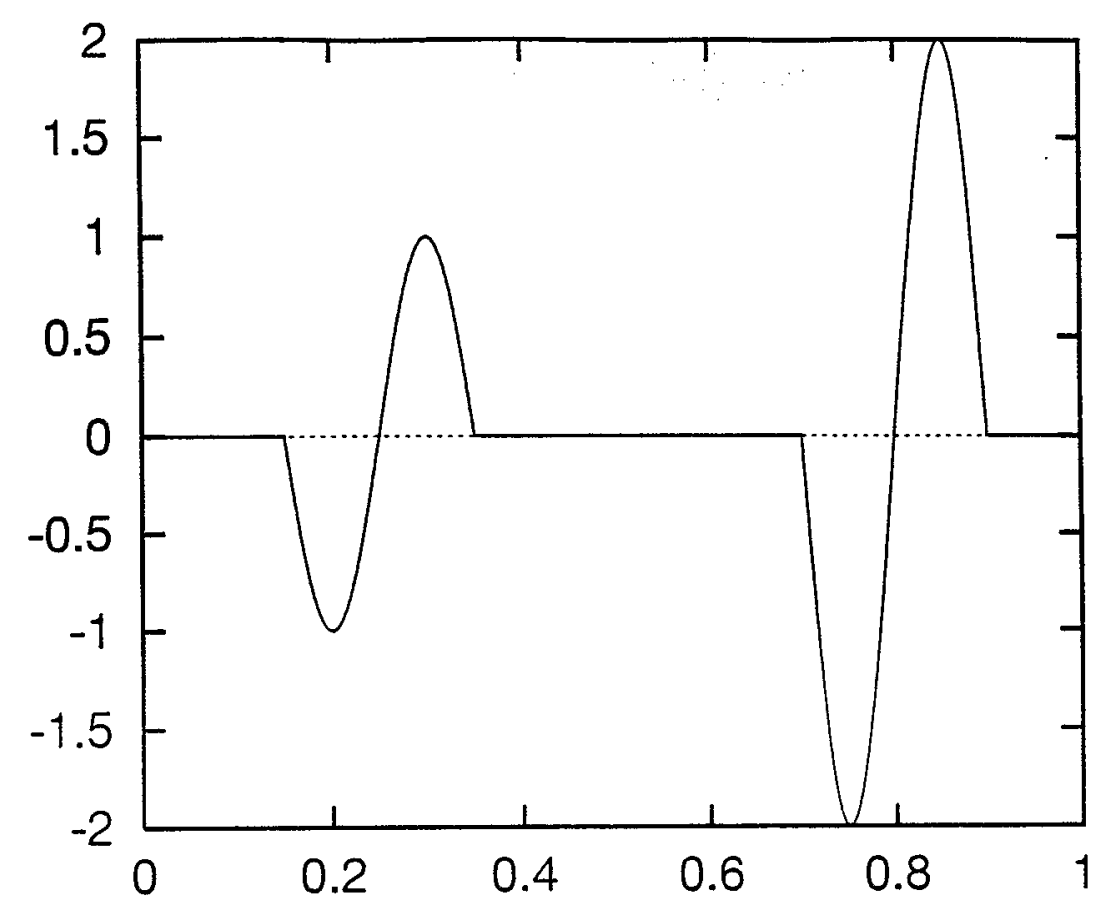

Figure 2: Example waveform for equation (13). The parameters are $\tau_{1}=$ $0.15, V_{1}=1, \tau_{2}=0.7$ and $V_{2}=2$. The revolution period was set to 1 and the half width to $w=0.1$.

where $[s]$ denotes the integer part of $s$ and the half width of the waveform, $w$ is fixed. Figure 2 is a canonical plot of $V(t)$ for a single revolution period. The model allows the centers and amplitudes to change for the next revolution. This model is quite general and a simpler version will be considered first.

Setting $V_{2} \equiv 0$ yields a model similar to the system used to create a gap in the coasting beam of the antiproton debuncher at Fermilab [1,2]. At Fermi the gap is used to accommodate the rise time of the kicker. In the AGS the gap must be long enough to accommodate the entire kicker pulse. The model dynamics as applied to the Booster and AGS are given by setting $\tau_{1} \equiv 0$ and varying $V_{1}$ in a saw-tooth pattern like that shown in Figure 3 . The cavity is on during the first injection, and the bunch is injected into the zero voltage region. The second Booster cycle is injected into the gap created by the cavity and $V_{1}$ is quickly dropped to zero. The energy spread in the beam after the first and second transfers are thus $\Delta E_{1}=\Delta E_{2}=\Delta E_{B}$ where $\Delta E_{B}$ is the energy spread in the Booster. On subsequent transfers the value of $V_{1}$ rises from zero and a gap is formed in the coasting beam. The next booster cycle is injected into this gap and $V_{1}$ is brought quickly to zero. An estimate of the increase in energy spread due to this process may be obtained by mixing the adiabatic and fast approximations. As $V_{1}$ rises the longitudinal extent of the beam is decreased fractionally by an amount $f \sim 1-2 w$, and the energy spread increases fractionally by $1 / f$. The next Booster fill is injected into the gap and $V_{1}$ is quickly brought to zero. Hence, 


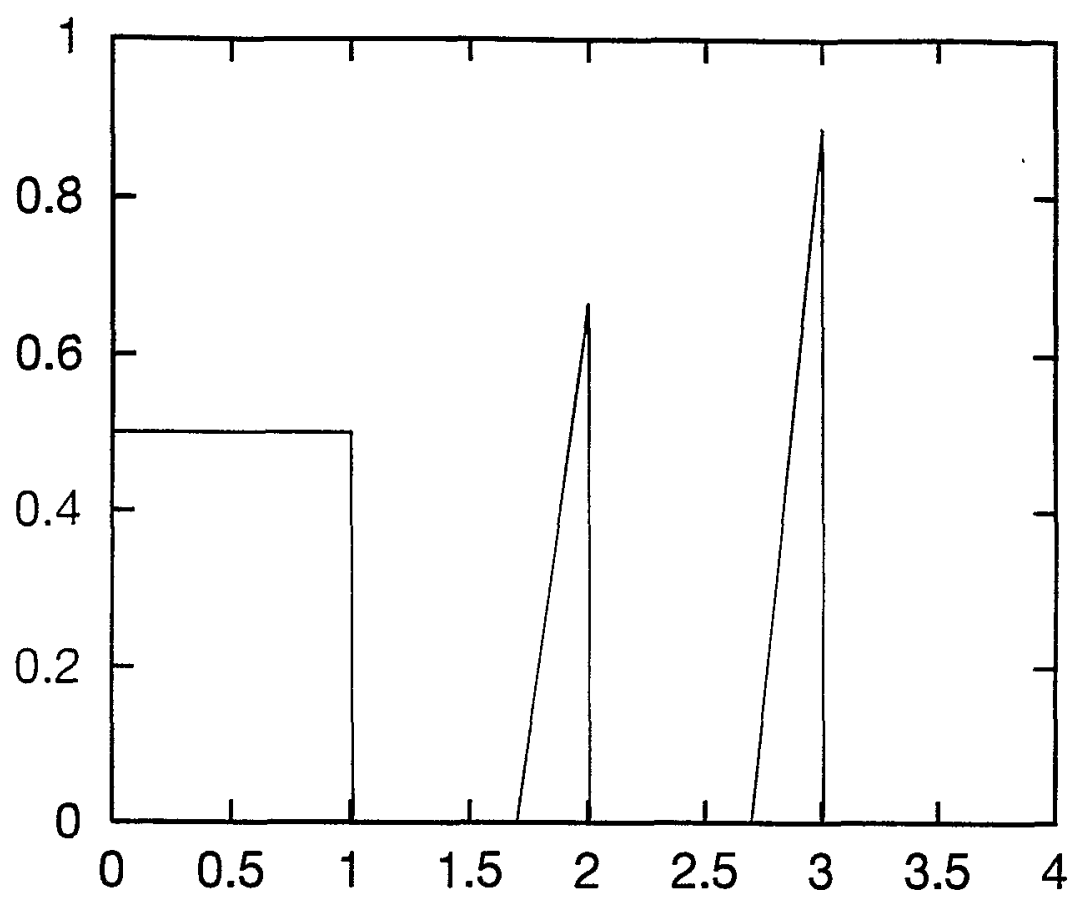

Figure 3: Example time dependence of the $V_{1}$ amplitude. The time between transfers has been set to unity and the first transfer occurs at $t=0$.

the energy spread will increase exponentially. For $f=0.8$ the energy spread in the AGS beam will be about $1.6 \Delta E_{B}$ after 4 transfers. Simulation results for this process are shown in Figure 4 which shows $\sqrt{H(\phi, E)}$ and $\sqrt{U(t, \phi)}$ for a swarm of particles just before the third Booster transfer of Figure 3. The square roots were chosen so that the physical area on the plot was close to the conserved phase space area occupied by the particles. It was assumed that bunching in the Booster would allow a gap of only $20 \%$ in the AGS and the simulation used equations (1) and (2). The cavity voltage rose from zero to its peak value in $20 \mathrm{~ms}$ and the initial energy spread of the beam was $\left|E-E_{0}\right| \leq E_{0} / 500$. The energy spread increased by a factor of 1.4, corresponding to $f=0.7$ and an effective gap length of 0.3 in the adiabatic approximation, which appears quite reasonable.

While a factor of two increase in energy spread is not too much of a problem for four transfers it is clear that the momentum aperture will quickly fill up if more than four transfers are used. Additionally, the peak value of $V_{1}$ will need to increase as more transfers are added, giving another limit. These problems can be partially overcome by using the second voltage component.

For case of two voltage components, the fast change in the voltage amplitude becomes unnecessary and preliminary results indicate that the longitudinal emittance can be nearly conserved.

Figure 5 shows simulation results for a possible scenario. To make the connection with adiabatic invariants more visible $\sqrt{H(\phi, E)}$ and $\sqrt{U(t, \phi)}$ are shown. Figure 5a shows four Booster cycles worth of particles outside 


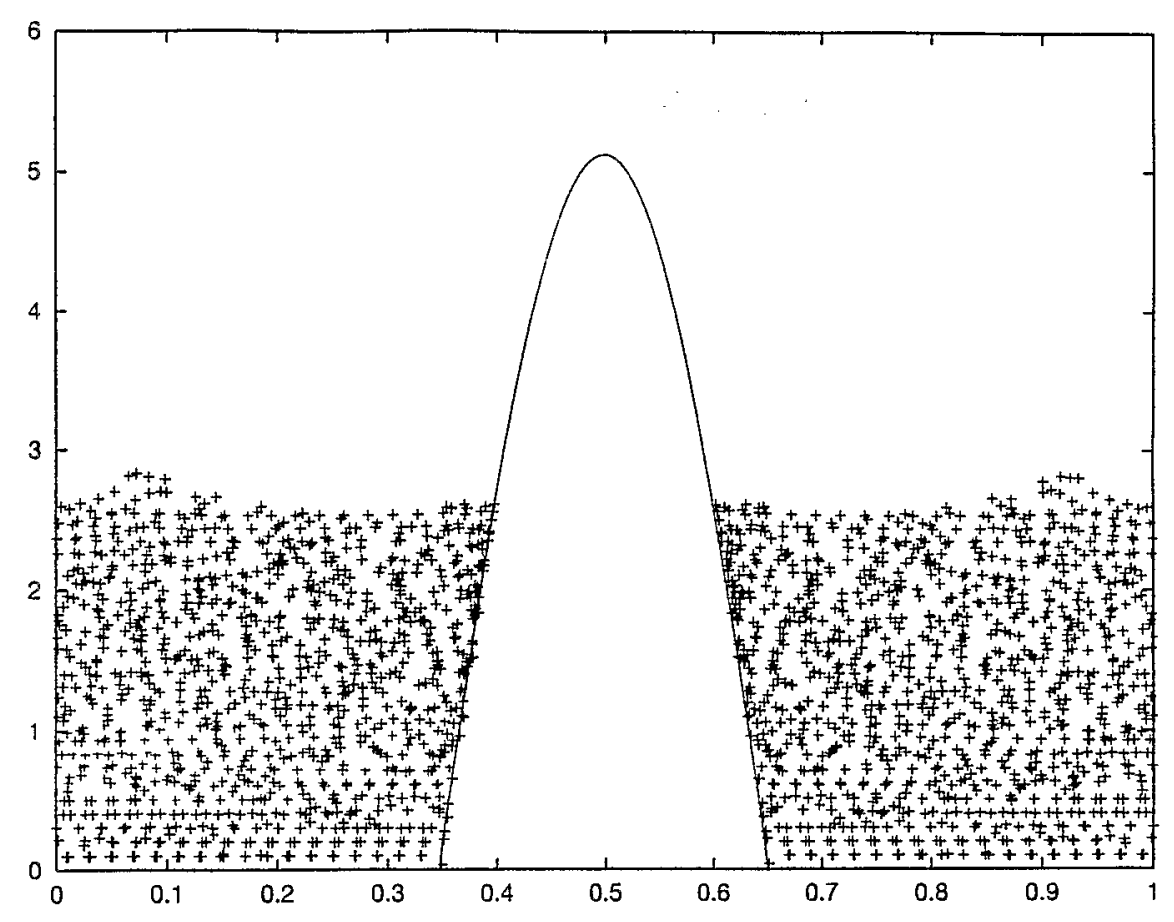

Figure 4: Simulation results for 2000 test particles with $f \approx 0.7$.

the barrier bucket and another Booster cycle has just been injected. In Figure $5 \mathrm{~b}$ the injected particles have been squeezed yielding a similar energy distribution for particles inside an outside the bucket. In Figure 5c the second voltage component has collapsed. In $5 \mathrm{~d}$ this component has returned in phase with the first. In Figures $5 \mathrm{e}$ and $\mathrm{f}$ the voltage components separate and grow making room for the next Booster cycle. In plot units, the initial peak momentum is 4 while the final peak momentum is 5 and the number of particles outside the barrier increases in the same ratio. There is very little emittance dilution during the process.

\section{Collective Effects}

For proton operation the main purpose of installing a barrier cavity would be to reduce the slow losses which seriously limit the amount of beam surviving to be accelerated. It has been observed that these losses are drastically reduced when the RF is turned off. More generally, reducing the peak current via longitudinal dilution reduces the slow loss. A barrier cavity system is a natural extension of this process and, ostensibly, will reduce the slow losses even further since the bunching factor will be increased to $I_{\text {avg }} / I_{\text {peak }} \sim 0.7$.

However, new effects arise because the particles now have a distribution of synchrotron tunes going from zero up to a maximum value that is roughly proportional to the energy spread in the beam. The simplest approximation is to neglect the walls of the barrier altogether and consider a coasting beam 

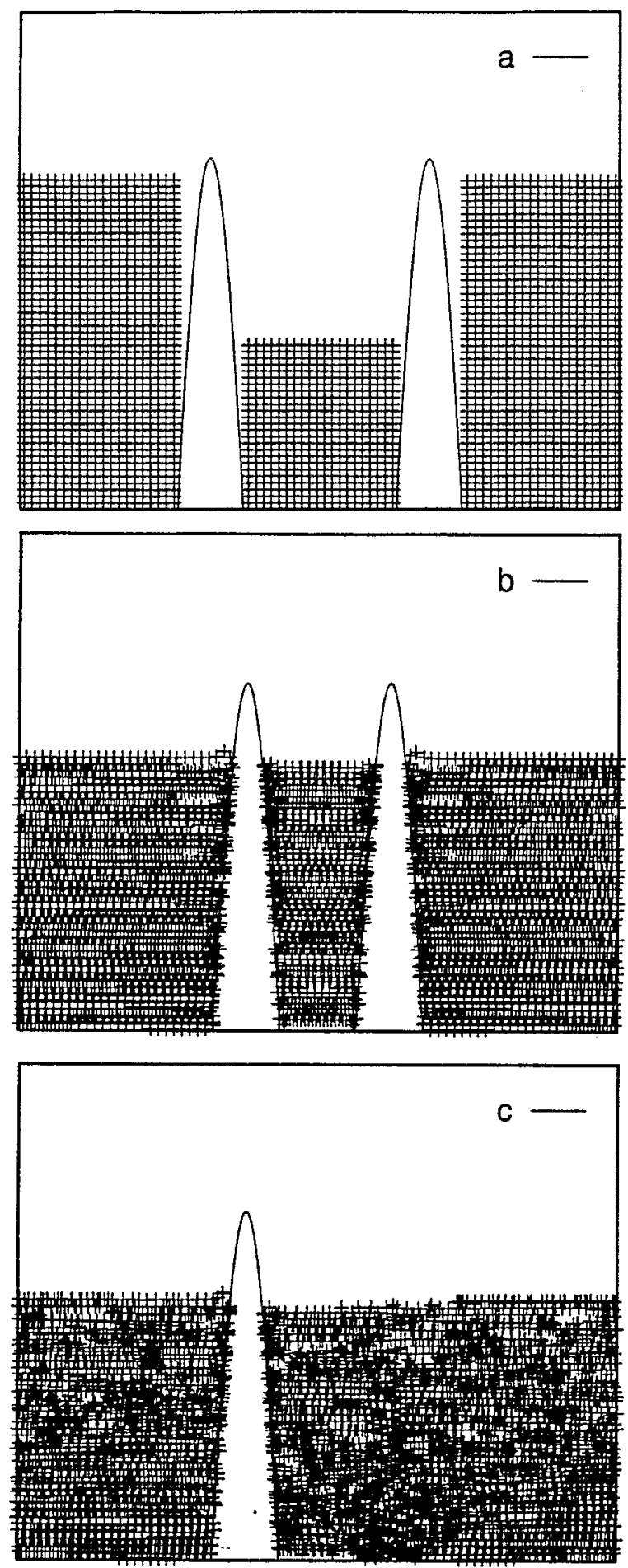
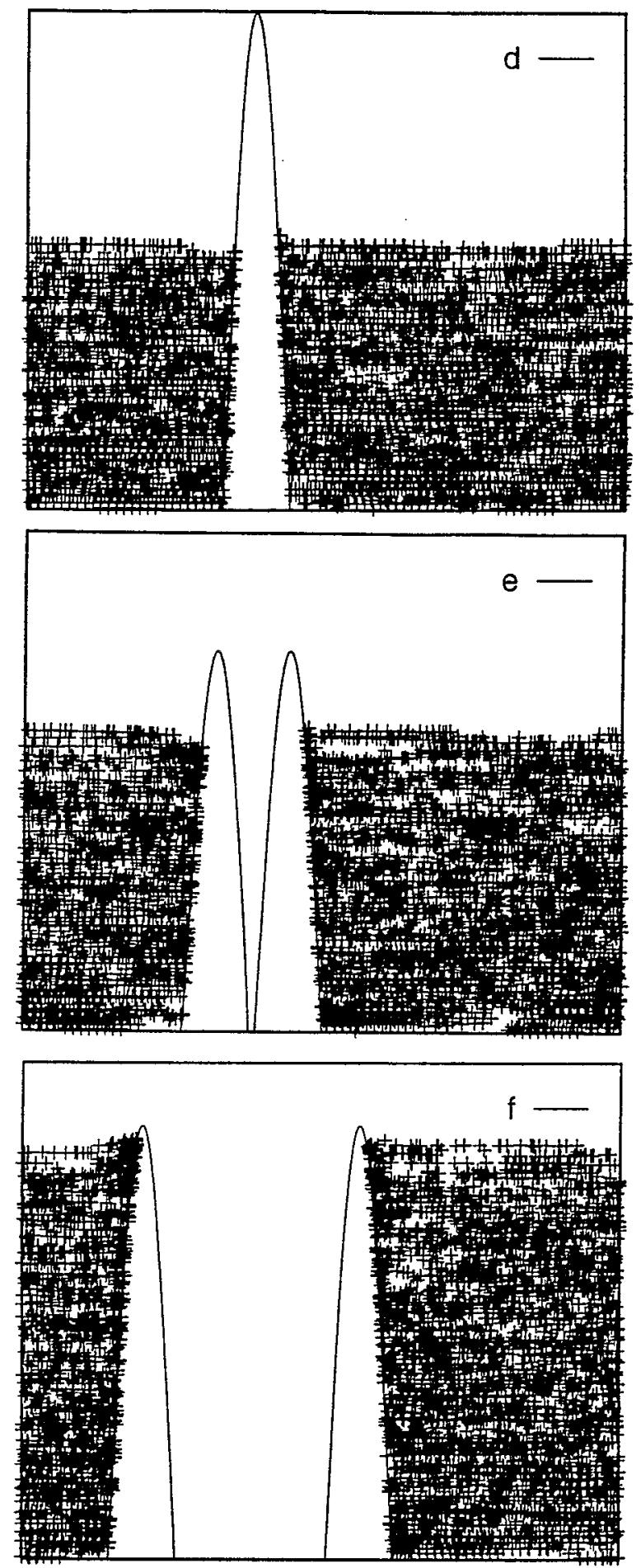

Figure 5: Simulation results for 5,000 test particles with two voltage components. The time interval between plots was $22 \mathrm{~ms}$ for the first 5 while the interval between plots e and $\mathrm{f}$ was $44 \mathrm{~ms}$. Note that $\sqrt{H(\phi, E)}$ and $\sqrt{U(t, \phi)}$ are plotted and that the axes for each subplot are the same. 
with the same energy spread and peak current. The coasting beam stability formulas are standard [3]. For no longitudinal instability the criterion is

$$
I_{\text {peak }}\left|\frac{Z_{\|}}{n}\right| \lesssim \frac{|\eta| \Delta E^{2}}{\beta_{0}^{2} E_{0} q}
$$

where $\Delta E$ is the full width at half maximum energy spread. For transverse instabilities the system will be stable if

$$
I_{p e a k} R\left|Z_{\perp}\left(\omega_{0}(Q-n)\right)\right| \lesssim \frac{2 \pi Q \Delta E}{\beta q}|\eta||n-Q+Q \xi / \eta|,
$$

and the inequality needs to be satisfied for $n>Q$. Note that a negative (positive) chromaticity helps damp the system below (above) transition as in the bunched beam case.

To apply equations (14) and (15) to the AGS several parameters are needed. For a working set assume protons with:

1) $\eta=-0.13$

2) $\xi=-0.5$

3) $Q=8.8$

4) $I_{\text {peak }}=3 \mathrm{~A}$

5) $\Delta E / E_{0}=0.01$

Using equations (14) and (15) the impedance limits are given by, $\left|Z_{\|} / n\right| \lesssim$ $12 \mathrm{k} \Omega$ and $\left|Z_{\perp}\right| \lesssim 17 \mathrm{M} \Omega / \mathrm{m}$. For the RF system currently in the AGS the undamped impedance of the fundamental is $8 \mathrm{k} \Omega$ but this is reduced to $\sim 1 \mathrm{k} \Omega$ using feedback. The longitudinal space charge impedance is of order $100 \Omega$ and the broad band impedance is even smaller. The transverse space charge impedance is of order $100 \mathrm{M} \Omega / \mathrm{m}$, so transverse instabilities might occur. Note that theory also predicts a strong transverse instability on the AGS porch for the current injection scheme. Using \pm 10 synchrotron modes I find a growth rate $70 \mathrm{~s}^{-1}$ for a chromaticity of -0.5 and $40 \mathrm{Tp}$ in the AGS. This is just the low frequency resistive wall instability and there is no reason to expect that the situation will be worsened by a barrier cavity system.

\section{References}

[1] J.E. Griffin, C. Ankenbrandt, J.A. MacLachlan, A. Moretti, IEEE TNS, 30, p. 3502, 1983. 
[2] V.K. Bharadwaj, J.E. Griffin, D.J. Harding, J.A. MacLachlan, IEEE TNS, 34, p. 1025, 1987.

[3] J.L. Laclare, CERN 85-19, p377, 1985. 